\title{
Accelerating tube-based model predictive control by constraint removal
}

\author{
Michael Jost, Gabriele Pannocchia and Martin Mönnigmann
}

\begin{abstract}
Tube-based model predictive control (MPC) is a variant of MPC that is suitable for constrained linear systems subject to additive bounded disturbances. We extend constraint removal, a technique recently introduced to accelerate nominal MPC, to tube-based MPC. Constraint removal detects inactive constraints before actually solving the MPC problem. By removing constraints that are known to be inactive from the optimization problem, the computational time required to solve it can be reduced considerably. We show that the number of constraints to be considered in the optimization problem decreases along any trajectory of the closed-loop system, until only the unconstrained optimization problem remains. The proposed variant of constraint removal is easy to apply. Since it does not depend on details of the optimization algorithm, it can easily be added to existing implementations of tube-based MPC.
\end{abstract}

\section{INTRODUCTION}

Model predictive control (MPC) is a popular method for the control of constrained multi-input multi-output systems. MPC builds on the fact that for nominal systems feedback and open-loop control are equivalent. As a consequence, the optimal control action can be found by solving an open-loop optimal control problem [1], [2]. However, if the system under consideration is subject to disturbances this equivalence does not hold, and the solution of the nominal problem is no longer optimal [2], [3].

Several approaches have been developed to overcome this limitation, see e.g. [4], [5] for surveys. The authors in [3], [6], for example, suggest to ignore the disturbance and rely on the inherent robustness of nominal MPC. Min-max MPC is based on minimizing a series of control laws over the worst case disturbance [2], [7]. In tube-based MPC a series of linear control laws is used to keep the state of the real system in a tube around the reference trajectory of the nominal system [1], [3], [8], [9].

We present a method to reduce the computational effort required in tube-based MPC. Our method is based on detecting inactive constraints before solving the MPC problem, removing these constraints, and saving computational time by solving the reduced optimization problem. Specifically, we use a variant of constraint removal that relies on the decrease of the cost function, which serves as a Lyapunov function. As opposed to earlier variants of constraint removal [10]-[12], Lyapunov function-based constraint removal is particularly easy to implement and does not require costly preparatory calculations. The proposed acceleration technique has been

M. Jost and M. Mönnigmann are with Automatic Control and Systems Theory, Department of Mechanical Engineering, RuhrUniversität Bochum, Germany. G. Pannocchia is with Department of Civil and Industrial Engineering, University of Pisa, Italy. E-mail: michael.s.jost@rub.de, gabriele.pannocchia@unipi.it, martin.moennigmannerub.de. presented in [13] for the first time to the knowledge of the authors. It is the point of the present paper to extend this technique to tube-based MPC.

The paper is organized as follows. We start with summarizing notation and preliminaries and concisely state the problem in Section II. In Section III we present the main result of this paper, i.e. the constraint removal technique and the formulation of the reduced, equivalent finite-horizon optimal control problem. An example is discussed in Section IV. The paper closes with conclusions and a brief outlook in Section V.

\section{Notation and Preliminaries}

Let $\oplus$ and $\ominus$ denote set addition and subtraction, respectively, i.e., for $A \subset \mathbb{R}^{n}$ and $B \subset \mathbb{R}^{n}$ we have $A \oplus B=$ $\{a+b \mid a \in A, b \in B\}$ and $A \ominus B=\{a \mid a \oplus B \subseteq A\}$. For any vector $x \in \mathbb{R}^{n},\|x\|$ denotes the Euclidean norm of $x$. For any set $\mathcal{S}$ let $|\mathcal{S}|$ denote its cardinality. If $M \in \mathbb{R}^{n \times n}$ is a positive definite matrix, we write $M \succ 0$. We denote the identity matrix in $\mathbb{R}^{n}$ by $I^{n \times n}$. Finally, we summarize some properties on ellipsoids that are required in Section III.

Lemma 1 Let $M \in \mathbb{R}^{s \times s}$ be a symmetric positive definite matrix, and consider any $\xi \in \mathbb{R}^{s}$ and $\alpha \geq 0$. Then $\xi^{\prime} M \xi \leq$ $\alpha^{2}$ implies $\|\xi\| \leq \frac{\alpha}{\sqrt{\lambda_{\min }(M)}}$.

Proof. First note $\lambda_{\min }(M) \xi^{\prime} \xi \leq \xi^{\prime} M \xi$ (see e.g. [14, Lemma 8.4.3]). Therefore $\xi^{\prime} M \xi \leq \alpha^{2}$ implies $\lambda_{\min }(M) \xi^{\prime} \xi \leq \alpha^{2}$. Since $M \succ 0$, we have $\lambda_{\min }(M)>0$, therefore $\sqrt{\lambda_{\min }(M)}$ is meaningful and the claim holds.

\section{PRoblem Formulation}

We consider constrained linear discrete-time state space systems subject to an additive (unknown) disturbance

$$
x_{k+1}=A x_{k}+B u_{k}+d_{k},
$$

where $x \in \mathbb{R}^{n}$ is the state, $u \in \mathbb{R}^{m}$ is the input, $d \in \mathbb{R}^{n}$ is the disturbance and $(A, B)$ with $A \in \mathbb{R}^{n \times n}, B \in \mathbb{R}^{n \times m}$ is stabilizable. We assume constraints

$$
u_{k} \in \mathbb{U}, \quad x_{k} \in \mathbb{X}
$$

apply for all $k$, where $\mathbb{U}$ and $\mathbb{X}$ are compact (closed and bounded) polyhedral sets that contain the origin as an interior point. Moreover, we assume

$$
d_{k} \in \mathbb{D}
$$

for all $k$, where $\mathbb{D}$ is a compact polyhedral set containing the origin in its interior.

It proves useful to define the nominal system

$$
\hat{x}_{k+1}=A \hat{x}_{k}+B \hat{u}_{k} .
$$


Nominal MPC builds on repeatedly calculating the optimal control sequence

$$
\mathbf{u}=\left[\hat{u}_{0}, \ldots, \hat{u}_{N-1}\right]
$$

for the system (4) by solving an open-loop optimal control problem with finite horizon $N$, and applying the first element $\hat{u}_{0}$. Tube-based MPC counteracts the disturbance in (1) by repeatedly calculating a sequence of control laws

$$
\mathbf{u}(x)=\left[\mu_{0}(x), \ldots, \mu_{N-1}(x)\right]
$$

instead of the control signals (5), and applying the first control law to (1). While the control sequence (5) results from a finite-dimensional optimal control problem, finding (6) requires to solve an infinite-dimensional problem. Since this problem is typically too complex [1], [3], the sequence of control laws (6) is replaced by a sequence of linear control laws

$$
\mu_{i}\left(x_{k}\right)=\hat{u}_{k+i}+K\left(x_{k+i}-\hat{x}_{k+i}\right), \quad i=0, \ldots, N-1
$$

In (7) $K \in \mathbb{R}^{m \times n}$ is a state feedback controller that is assumed to stabilize the nominal closed-loop system $\hat{x}_{k+1}=$ $A_{K} \hat{x}_{k}, A_{K}=A+B K$. In other words, $A_{K}$ must only have eigenvalues strictly inside the unit disc.

Since the system (1) is subject to a disturbance $d(k)$, it will not remain at the origin if a controller drives it there. The best we can achieve is to keep the system in a robust positively invariant (RPI) set that contains the origin. More formally, if $\mathbb{Z} \subset \mathbb{R}^{n}$ is such an RPI set, then we have $A_{K} \mathbb{Z} \oplus \mathbb{D} \subseteq \mathbb{Z}$ by definition of $\mathbb{Z}$ [1]. Since we want the state of the closed-loop system to be as close as possible to the origin, $\mathbb{Z}$ should be as small as possible. The minimal RPI set can be constructed for special cases [1], [15], for example, if $A_{K}$ is nilpotent. In general, the minimal RPI set can only be approximated conservatively. An iterative method for the construction of a positively invariant outer approximation of the minimal RPI set is provided in [15].

The constraints (2) need to be tightened in order to guarantee their satisfaction in the presence of disturbances [1]. This yields

$$
\hat{\mathbb{U}}=\mathbb{U} \ominus K \mathbb{Z}, \quad \hat{\mathbb{X}}=\mathbb{X} \ominus \mathbb{Z} .
$$

Note that $\hat{\mathbb{U}}$ and $\hat{\mathbb{X}}$ are required to contain the origin in their interior despite the tightening. Roughly speaking, this condition is fulfilled if the constraints (2) are not too restrictive and the disturbance set is relatively small.

Tube-based MPC calculates the optimal sequence of control policies of the form (7) by solving the quadratic program

$$
\begin{array}{ll} 
& V^{\star}(x)=\min _{\hat{\mathbf{x}}, \hat{\mathbf{u}}} \ell_{f}\left(\hat{x}_{N}\right)+\sum_{i=0}^{N-1} \ell\left(\hat{x}_{i}, \hat{u}_{i}\right) \\
\text { s.t. } & \hat{x}_{i+1}=A \hat{x}_{i}+B \hat{u}_{i}, \quad i=0, \ldots, N-1, \\
& \hat{x}_{i} \in \hat{\mathbb{X}}, \quad i=0, \ldots, N-1, \\
& \hat{u}_{i} \in \hat{\mathbb{U}}, \quad i=0, \ldots, N-1, \\
& \hat{x}_{N} \in \hat{\mathbb{X}}_{f} \\
& x \in \hat{x}_{0} \oplus \mathbb{Z} .
\end{array}
$$

where $\hat{\mathbf{u}}=\left[\hat{u}_{0}, \ldots, \hat{u}_{N-1}\right], \hat{\mathbf{x}}=\left[\hat{x}_{0}, \ldots, \hat{x}_{N-1}\right], \ell_{f}\left(\hat{x}_{N}\right)=$ $\frac{1}{2} \hat{x}_{N}^{\prime} P \hat{x}_{N}$ is the terminal cost function with weighting matrix $\stackrel{P}{P} \mathbb{R}^{n \times n}, P \succ 0, \ell(\hat{x}, \hat{u})=\frac{1}{2}\left(\hat{x}^{\prime} Q \hat{x}+\hat{u}^{\prime} R \hat{u}\right)$ is the stage cost with $Q \in \mathbb{R}^{n \times n}, Q \succ 0$ and $R \in \mathbb{R}^{m \times m}, R \succ 0, N$ is the problem horizon and $\hat{\mathbb{X}}_{f} \subset \hat{\mathbb{X}}$ is a positively invariant terminal set. We note for later use that the constraints in (8) define the set

$$
\mathcal{U}_{N}(x)=\left\{\hat{\mathbf{u}} \in \mathbb{R}^{m N} \mid \exists \hat{\mathbf{x}} \text { such that (8b)-(8e) hold }\right\} .
$$

We refer by $\hat{\mathbf{u}}^{\star}(x)$ and $\hat{\mathbf{x}}^{\star}(x)$ to the optimal solution of (8) and by $\hat{u}_{0}^{\star}(x)$ and $\hat{x}_{0}^{\star}(x)$ to the first $m$ elements of $\hat{\mathbf{u}}^{\star}(x)$ and the first $n$ elements of $\hat{\mathbf{x}}^{\star}(x)$, respectively. Moreover, we denote by $V^{\star}(x)$ to the optimal value function.

Using the optimal solution obtained from solving (8) together with (7) results in the feedback law

$$
\kappa(x)=\hat{u}_{0}^{\star}(x)+K\left(x-\hat{x}_{0}^{\star}(x)\right),
$$

which implements the control sequence $\hat{u}_{0}^{\star}(x)$ resulting for the nominal MPC problem and the weighted error between the true and the nominal system state $K\left(x-\hat{x}_{0}^{\star}(x)\right)$. Roughly speaking, the first part $\hat{u}_{0}^{\star}(x)$ steers the nominal system towards the origin while the weighted error $K\left(x-\hat{x}_{0}^{\star}(x)\right)$ keeps the trajectory of the real system close to the trajectory of the nominal system.

It was shown in [1] that the objective function $V^{\star}(x)$ of (8) is strictly decreasing along any trajectory of the true system (1) with control law (9), and zero within the RPI set $\mathbb{Z}$. More formally, it can be shown that there exists a strictly positive constant $c_{1}$ such that

$$
\begin{aligned}
& V^{\star}\left(x^{+}\right)-V^{\star}(x) \leq-c_{1}\left\|\hat{x}_{0}^{\star}(x)\right\|_{2}^{2}, \\
& \text { for all } x \in \mathbb{X} \backslash \mathbb{Z}, x^{+}=A x+B \kappa(x)+d, d \in \mathbb{D} \\
& \qquad V^{\star}(x)=0, \text { for all } x \in \mathbb{Z},
\end{aligned}
$$

Notice that $x^{+}=A x+B \kappa(x)+d$ denotes any admissible successor state that results from applying the control law (9) to system (1). Furthermore, $V^{\star}(x)=0$ for all $x \in \mathbb{Z}$ because it follows that $\hat{x}_{0}^{\star}(x)=0$. Similarly, it follows that $V^{\star}(x)>$ 0 for all $x \in \mathbb{X} \backslash \mathbb{Z}$ because of (8f).

Thus, as proven in [1, Theorem 1, p. 222], $V^{\star}(x)$ is a robust Lyapunov function of the closed-loop system $x_{k+1}=$ $A x_{k}+B \kappa\left(x_{k}\right)+d_{k}$, and the set $\mathbb{Z}$ is robustly exponentially stable with region of attraction

$$
\mathcal{X}=\left\{x \in \mathbb{R}^{n} \mid \exists \hat{x}_{0} \text { such that } x \in \hat{x}_{0} \oplus \mathbb{Z}, \mathcal{U}_{N}(x) \neq \emptyset\right\},
$$

where $d_{k} \in \mathbb{D}$ for all $k$.

As a final preparation, we recall that the optimization problem (8) can be rewritten as

$$
\begin{aligned}
V^{\star}(x)=\min _{\hat{\mathbf{z}}} & \frac{1}{2} \hat{\mathbf{z}}^{\prime} \Gamma \hat{\mathbf{z}} \\
\text { s.t. } & G \hat{\mathbf{z}} \leq w(x),
\end{aligned}
$$

by substituting the states $\hat{x}_{1}, \ldots, \hat{x}_{N}$. In (11), $\hat{\mathbf{z}}^{\prime}=\left[\hat{\mathbf{u}}^{\prime}, \hat{x}_{0}^{\prime}\right]$, $\Gamma \in \mathbb{R}^{m N+n \times m N+n}, G \in \mathbb{R}^{q \times m N+n}$ and $w: \mathbb{R}^{n} \rightarrow \mathbb{R}^{q}$ is an affine function of the state. It can be shown that $\Gamma$ is a positive definite matrix. This property is a direct consequence of the fact that (8) is a strictly convex optimization problem 
under the assumptions stated above (namely, $R \succ 0$ and $Q \succ 0)$.

We call the $i$-th constraint of (11) inactive at the optimum if $G^{i} \hat{\mathbf{z}}^{\star}(x)<w^{i}(x)$, and active if $G^{i} \hat{\mathbf{z}}^{\star}(x)=w^{i}(x)$. The sets of inactive and active constraints for an arbitrary but fixed state $x \in \mathcal{X}$ are denoted by

$$
\begin{aligned}
& \mathcal{I}(x)=\left\{i \in \mathcal{Q} \mid G^{i} \hat{\mathbf{z}}^{\star}(x)<w^{i}(x)\right\}, \\
& \mathcal{A}(x)=\left\{i \in \mathcal{Q} \mid G^{i} \hat{\mathbf{z}}^{\star}(x)=w^{i}(x)\right\},
\end{aligned}
$$

respectively, where $\mathcal{Q}=\{1, \ldots, q\}$. For later use we note that the solution of the unconstrained optimization problem (11) is given by

$$
\hat{\mathbf{z}}^{\star}(x)=0,
$$

which immediately follows from the optimality conditions. Since $\hat{\mathbf{z}}^{\prime}=\left[\hat{\mathbf{u}}^{\prime}, \hat{x}_{0}^{\prime}\right]$ by definition, (13) implies $\hat{u}_{0}^{\star}(x)=0$ and $\hat{x}_{0}^{\star}(x)=0$, and the optimal control law (9) reduces to $\kappa(x)=K x$. In other words, the robust state feedback controller (9) is used to keep the system state inside the RPI set $\mathbb{Z}$ if no constraints are active.

\section{Constraint REMOVAl In TUBE-BASED MPC}

In this section we show how the number of constraints of the optimization problem (11) can be reduced by identifying constraints that are proved to be inactive at the optimal solution before solving (11).

First, we show how inactive constraints can be identified based on an upper bound on the optimal solution to (11).

Lemma 2 Let $x \in \mathcal{X}$ and $c \in \mathbb{R}, c>0$. Moreover, assume that $\left\|\hat{\mathbf{z}}^{\star}\left(x^{+}\right)\right\| \leq c$ holds. Then $\left\|G^{i}\right\| c<w^{i}(x) \Rightarrow i \in \mathcal{I}(x)$.

Proof. Consider the relations

$$
G^{i} \hat{\mathbf{z}}^{\star}(x) \leq\left|G^{i} \hat{\mathbf{z}}^{\star}(x)\right| \leq\left\|G^{i}\right\|\left\|\hat{\mathbf{z}}^{\star}(x)\right\| \leq\left\|G^{i}\right\| c,
$$

where $G^{i} \hat{\mathbf{z}}^{\star}(x) \leq\left|G^{i} \hat{\mathbf{z}}^{\star}(x)\right|$ holds since $\xi \leq|\xi|$ for all $\xi \in$ $\mathbb{R}$, where $\left|G^{i} \hat{\mathbf{z}}^{\star}(x)\right| \leq\left\|G^{i}\right\|\left\|\hat{\mathbf{z}}^{\star}(x)\right\|$ holds due to Cauchy and Schwarz's inequality, and where $\left\|G^{i}\right\|\left\|\hat{\mathbf{z}}^{\star}(x)\right\| \leq\left\|G^{i}\right\| c$ holds by assumption. Combining (14) and $\left\|G^{i}\right\| c<w^{i}(x)$ implies $G^{i} \hat{\mathbf{z}}^{\star}(x)<w^{i}(x)$, which yields $i \in \mathcal{I}(x)$ by definition of $\mathcal{I}(x)$.

If a subset of constraints $\mathcal{J}(x) \subseteq \mathcal{Q}$ is known to be inactive before solving the optimization problem (11), these constraints can be removed from consideration, since they play no role at the optimum. We summarize this in the following lemma and show how to create a reduced quadratic program, which has the same solution as (11) but is smaller in size.

Lemma 3 Let $x \in \mathcal{X}$ be arbitrary. Consider a set $\mathcal{J}(x) \subseteq$ $\mathcal{Q}$, where it is assumed that $\left\|G^{i}\right\| c<w^{i}(x), c>0$ holds for every $i \in \mathcal{J}(x)$. Then both the reduced quadratic program

$$
\begin{aligned}
\min _{\hat{\mathbf{z}}} & \frac{1}{2} \hat{\mathbf{z}}^{\prime} \Gamma \hat{\mathbf{z}} \\
\text { s.t. } & G^{i} \hat{\mathbf{z}} \leq w^{i}(x), \quad i \in \mathcal{Q} \backslash \mathcal{J}(x),
\end{aligned}
$$

and (11) have the same unique solution $\hat{\mathbf{z}}^{\star}(x)$.
We state Lemma 3 without proof. Lemma 3 can easily be proven by showing that the same set of KKT conditions results for both (11) and (15), and by setting the Lagrange multipliers $\lambda_{i}, i \in \mathcal{J}(x)$, to zero in the KKT conditions resulting for (11). The corresponding statement for the nominal case was proven in [12].

\section{A. A priori bounds on $\left\|\hat{\mathbf{z}}^{\star}\right\|$}

An upper bound $c$ on the optimal solution, $\left\|\hat{\mathbf{z}}^{\star}\right\| \leq c$, is required in Lemma 2. We show how to compute such a bound in this section. Specifically, we would like to obtain such a bound for the successor state $x^{+}$, i.e. $c$ such that $\left\|\hat{\mathbf{z}}^{\star}\left(x^{+}\right)\right\| \leq$ $c$, before solving the MPC problem for $x^{+}$. It turns out the desired bound can be derived from the solution to the MPC problem from the current state. First, we state in Lemma 4 that $\hat{\mathbf{z}}^{\star}\left(x^{+}\right)$lies in an ellipsoid that can easily be calculated before solving the MPC problem for $x^{+}$(Lemma 4). This ellipsoid implies the desired bound (Corollary 5). Secondly, we show in Lemma 6 how a simple upper bound can be constructed from the constraints directly. This second bound is independent of the state and therefore can be determined before runtime of the MPC controller.

Lemma 4 Let $x \in \mathcal{X}$ be arbitrary and assume the optimal solution $\hat{\mathbf{z}}^{\star}(x)$ for the current state $x$ has already been calculated. Then, the optimal input sequence $\hat{\mathbf{z}}^{\star}\left(x^{+}\right)$for the successor state $x^{+}$lies in the ellipsoid

$$
\mathcal{E}(x)=\left\{\mathbf{z} \in \mathbb{R}^{m N+n} \mid \frac{1}{2} \mathbf{z}^{\prime} \Gamma \mathbf{z} \leq \rho(x)\right\}
$$

where

$$
\rho(x)=V^{\star}(x)
$$

is strictly positive for all $x \in \mathcal{X} \backslash \mathbb{Z}$ and $\rho(x)=0, \forall x \in \mathbb{Z}$.

Proof. First note that $\rho(x)>0$ for all $x \in \mathcal{X} \backslash \mathbb{Z}$ and $\rho(x)=0$ for all $x \in \mathbb{Z}$ holds according to (10). Recalling that $\Gamma$ is symmetric and positive definite, i.e., $\Gamma^{\prime}=\Gamma, \Gamma \succ 0$, it follows that (16) defines an ellipsoid in the augmented space (of inputs and initial nominal state).

It remains to show that $\hat{\mathbf{z}}^{\star}\left(x^{+}\right) \in \mathcal{E}(x)$. Consider the relation $V^{\star}\left(x^{+}\right) \leq V^{\star}(x)$, which follows from (10). Substituting $V^{\star}\left(x^{+}\right)=\frac{1}{2} \hat{\mathbf{z}}^{\star}\left(x^{+}\right) \Gamma \hat{\mathbf{z}}^{\star}\left(x^{+}\right)$and using (17) yields

$$
\frac{1}{2} \hat{\mathbf{z}}^{\star}\left(x^{+}\right) \Gamma \hat{\mathbf{z}}^{\star}\left(x^{+}\right) \leq \rho(x),
$$

which is equivalent to the inequality in (16) and therefore proves $\hat{\mathbf{z}}^{\star}\left(x^{+}\right) \in \mathcal{E}$.

The following corollary states the upper bound on $\hat{\mathbf{z}}^{\star}\left(x^{+}\right)$ that results from $\hat{\mathbf{z}}^{\star}\left(x^{+}\right) \in \mathcal{E}(x)$.

Corollary 5 Let $x \in \mathcal{X}$ be arbitrary, assume the optimal solution $\hat{\mathbf{z}}^{\star}(x)$ for the current state $x$ has been found, and let $\rho(x)$ be defined as in (17). Then the norm of the optimal solution to (11) at the next time step, $\left\|\hat{\mathbf{z}}^{\star}\left(x^{+}\right)\right\|$, is bounded above by

$$
\tilde{z}(x)=\sqrt{\frac{2 \rho(x)}{\lambda_{\min }(\Gamma)}},
$$

i.e., $\left\|\hat{\mathbf{z}}^{\star}\left(x^{+}\right)\right\| \leq \tilde{z}(x)$ holds. 


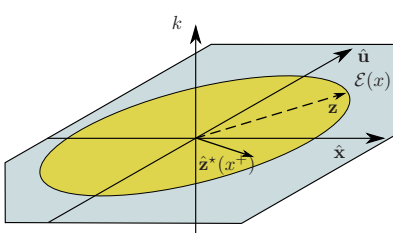

(a)

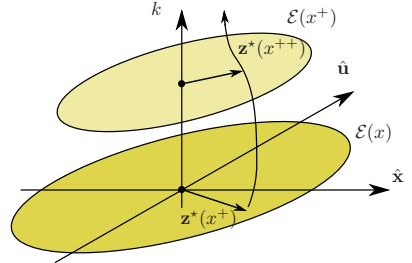

(b)
Fig. 1: Illustration of Lemma 4 and Corollary 5: (a) The optimal solution $\hat{\mathbf{z}}^{\star}\left(x^{+}\right)$to the quadratic program (11) lies in the ellipsoid $\mathcal{E}(x)$ centered at the origin. Thus, the largest semi-axes yields an upper bound on the vector of decision variables $\hat{\mathbf{z}}^{\star}\left(x^{+}\right)$. (b) The ellipsoids $\mathcal{E}(x), \mathcal{E}\left(x^{+}\right)$are scaled by the Lyapunov function $V^{\star}(x)$. Since $V^{\star}(x)$ decreases along any trajectory of the system, $\mathcal{E}\left(x^{+}\right)$shrinks along any trajectory. As a consequence, the number of constraints of the reduced optimization problem is nonincreasing along any trajectory of the closed-loop system.

Proof. Let $\mathbf{z} \in \mathcal{E}(x)$ be arbitrary. According to Lemma 1 this implies

$$
\|\mathbf{z}\| \leq \sqrt{\frac{2 \rho(x)}{\lambda_{\min }(\Gamma)}},
$$

where we used $\lambda_{\min }\left(\frac{1}{2} \Gamma\right)=\frac{1}{2} \lambda_{\min }(\Gamma)$, which proves the claim.

A graphical interpretation of Corollary 5, which shows the ellipsoid $\mathcal{E}(x)$ and the resulting upper bound $\tilde{z}(x)$ is given in Figure 1 . We stress again that Corollary 5 yields a bound on the optimal solution $\left\|\hat{\mathbf{z}}^{\star}\left(x^{+}\right)\right\|$for the successor state $x^{+}$ from information derived from the optimal solution $\left\|\hat{\mathbf{z}}^{\star}(x)\right\|$ at the current state $x$.

Finally, we derive a simple bound on $\left\|\hat{\mathbf{z}}^{\star}\left(x^{+}\right)\right\|$that is independent of $x$ and $x^{+}$. Since it does not depend on the state, this bound can be calculated offline. It therefore does not require any computation time during the runtime of the MPC controller. Moreover, it applies before the MPC problem is solved for the first time.

Lemma 6 Consider the optimal control problem (8). Let $\nu=\left(\nu^{1}, \ldots, \nu^{m}\right)$ and $\xi=\left(\xi^{1}, \ldots, \xi^{n}\right)$ where:

$$
\begin{array}{ll}
\nu^{l}=\arg \max _{u \in \hat{\mathbb{U}}}\left|u^{l}\right| & l=1, \ldots, m \\
\xi^{l}=\arg \max _{x \in \hat{\mathbb{X}}}\left|x^{l}\right| & l=1, \ldots, n
\end{array}
$$

Then, $\left\|\hat{\mathbf{z}}^{\star}(x)\right\|$ is bounded above by

$$
\bar{z}=\sqrt{N\|\nu\|^{2}+\|\xi\|^{2}},
$$

i.e., $\left\|\hat{\mathbf{z}}^{\star}(x)\right\| \leq \bar{z}$ holds for all $x \in \mathcal{X}$.

Proof. From $\hat{\mathbf{z}}=\left(\hat{u}_{0}^{\prime}, \ldots, \hat{u}_{N-1}^{\prime}, \hat{x}\right)^{\prime}$ we infer $\|\hat{\mathbf{z}}\|^{2}=$ $\sum_{i=0}^{N-1}\left\|\hat{u}_{i}\right\|^{2}+\|\hat{x}\|^{2}$. Since $\hat{u}_{i} \in \hat{\mathbb{U}}$, (20) implies $\left\|\hat{u}_{i}\right\|^{2} \leq$ $\sum_{l=1}^{m}\left(\nu^{l}\right)^{2}$ for all $i$. Likewise, since $\hat{x} \in \hat{\mathbb{X}}$, (21) implies $\|x\|^{2} \leq \sum_{l=1}^{n}\left(\xi^{l}\right)^{2}$. Thus, we have $\|\hat{\mathbf{z}}\|^{2} \leq N \sum_{l=1}^{m}\left(\nu^{l}\right)^{2}+$ $\sum_{l=1}^{n}\left(\xi^{l}\right)^{2}$, which proves the claim.

We note that (20) and (21) can be solved as linear programs (LP) in $m+2$ and $n+2$ variables, respectively. Moreover, those LPs need to be solved once and offline.

\section{B. Reduced MPC problem}

Finally, we show how the bounds introduced in Corollary 5 and Lemma 6 can be used to detect and remove inactive constraints in the MPC problem (15).

Lemma 7 Let $x, x^{+}, \rho(x), \hat{\mathbf{z}}^{\star}(x), \tilde{z}(x)$ be as in Corollary 5 , let $\bar{z}$ be as in Lemma 6 , and let

$$
\mathcal{J}\left(x^{+}\right)=\left\{i \in \mathcal{Q} \mid\left\|G^{i}\right\| \min (\tilde{z}(x), \bar{z})<w^{i}\left(x^{+}\right)\right\} .
$$

Then the optimal input sequence $\hat{\mathbf{z}}^{\star}\left(x^{+}\right)$for the closed-loop successor state $x^{+}$is the solution of the reduced $Q P$

$$
\begin{array}{ll}
\min _{\hat{\mathbf{z}}} & \frac{1}{2} \hat{\mathbf{z}}^{\prime} \Gamma \hat{\mathbf{z}} \\
\text { s.t. } & G^{i} \hat{\mathbf{z}} \leq w^{i}\left(x^{+}\right), \quad i \in \mathcal{Q} \backslash \mathcal{J}\left(x^{+}\right),
\end{array}
$$

Proof. Corollary 5 and Lemma 6 imply that $\left\|\hat{\mathbf{z}}^{\star}\left(x^{+}\right)\right\| \leq$ $\tilde{z}(x)$ and $\left\|\hat{\mathbf{z}}^{\star}\left(x^{+}\right)\right\| \leq \bar{z}$, respectively. Therefore, $\left\|\hat{\mathbf{z}}^{\star}\left(x^{+}\right)\right\| \leq \min (\tilde{z}(x), \bar{z})$. Consider any $i \in \mathcal{J}\left(x^{+}\right)$. Applying Lemma 2 to $x^{+}$for $c=\min (\tilde{z}(x), \bar{z})$ yields $i \in \mathcal{I}$ at $\hat{\mathbf{z}}^{\star}\left(x^{+}\right)$. The claim then follows from Lemma 3 .

If the optimization problem (23) is unconstrained, i.e. $\left|\mathcal{J}\left(x^{+}\right)\right|=q$, all constraints are inactive at the successor state $x^{+}$. Thus, the optimal solution is given by (13) and the successor state $x^{+}$lies within the set $\mathbb{Z}$. Since the set $\mathbb{Z}$ is an RPI set of the closed-loop system that satisfies $A_{K} \mathbb{Z} \oplus \mathbb{D} \subseteq \mathbb{Z}$, the state remains inside $\mathbb{Z}$ for all future time steps. We summarize this in the following remark.

Remark 8 If the $Q P$ is unconstrained for the state $x(\breve{k})$ at time $\breve{k}$, the unconstrained solution (13) is optimal for all $k \geq \breve{k}$.

An implementation of Lemma 7 is given in Algorithm 1. The algorithm shows how the reduced quadratic program can be constructed and solved for $\hat{\mathbf{z}}^{\star}\left(x^{+}\right)$at time step $x^{+}$ based on the value of the Lyapunov function $V^{\star}(x)$ at the previous time step $x$. If the reduced quadratic program is detected to be unconstrained, i.e., $\left|\mathcal{J}\left(x^{+}\right)\right|=q$ in line 11, the unconstrained solution $\kappa\left(x^{+}\right)=K x^{+}$can be applied

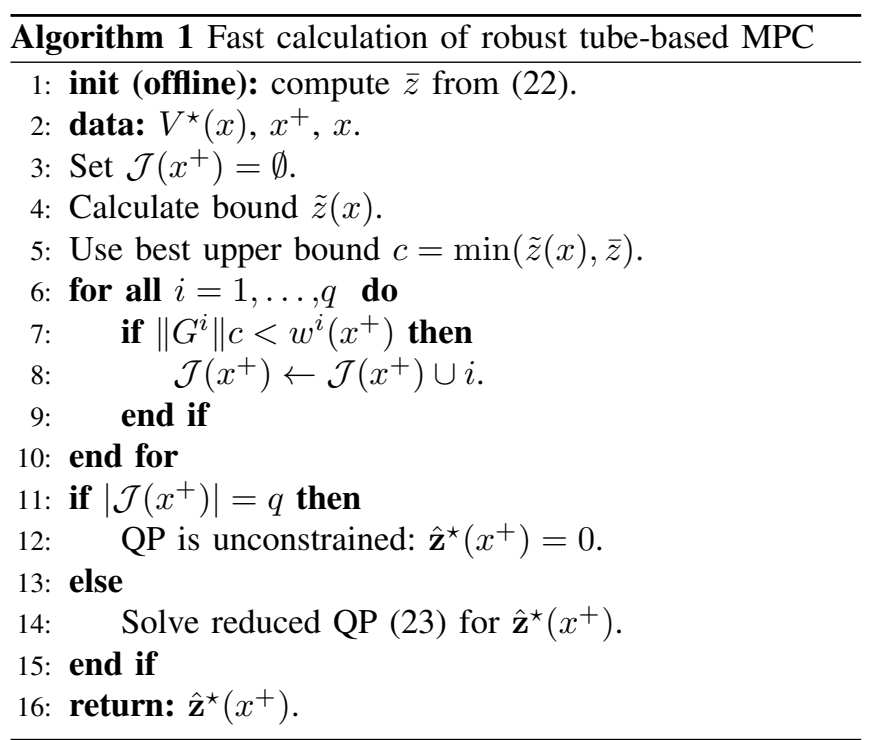


immediately (cf. line 12) and no optimization problem has to be solved. Otherwise, the reduced QP (23) is solved for the optimal $\hat{\mathbf{z}}^{\star}\left(x^{+}\right)$(cf. line 14). When solving the MPC problem for the first time, the bound $\tilde{z}(x)$ is not yet available, but $\bar{z}$ can be used.

\section{EXAMPLE}

We consider the discrete-time state space system that results from discretizing the continuous-time system with transfer function matrix

$$
G(s)=\left[\begin{array}{cc}
\frac{0.05}{36 s^{2}+6 s+1} & \frac{0.02(2 s+1)}{8 s+1} \\
\frac{0.02(2 s+1)}{8 s+1} & \frac{0.05}{12 s^{2}+3 s+1}
\end{array}\right]
$$

using zero-order hold with $T_{s}=1 \mathrm{~s}$. The resulting state space system of the form (1) has $n=6$ states and $m=2$ inputs. State space matrices are given in the appendix. We set the horizon, weighting matrices and the controller to $N=40$, $Q=10 I^{6 \times 6}, R=0.01 I^{2 \times 2}$, the solution to the discrete-time algebraic Riccati equation

$$
P=Q+A^{\prime} P A-A^{\prime} P B\left(R+B^{\prime} P B\right)^{-1} B^{\prime} P A,
$$

and

$$
K=-\left(R+B^{\prime} P B\right)^{-1} B^{\prime} P A,
$$

respectively. The state and input constraints (2) read (for all $k)$

$$
\begin{aligned}
-15 & \leq x^{i}(k) \leq 15, & & i=1, \ldots, 6, \\
-3 & \leq u^{i}(k) \leq 3, & & i=1,2 .
\end{aligned}
$$

Finally, the disturbance (3) is assumed to satisfy (for all $k$ )

$$
-0.01 \leq d^{i}(k) \leq 0.01, \quad i=1, \ldots, 6 .
$$

We used the algorithm presented in [15] to calculate an invariant approximation of the minimal robust positively invariant set $\mathbb{Z}$ with $\epsilon=1$ for $A+B K$. The terminal set $\mathbb{X}_{f}$ was calculated with the algorithm presented in [16]. The resulting QP to be solved during each time step has 840 constraints and 86 decision variables.

We calculated trajectories of the closed-loop system for 6250 random initial states for 40 time steps each and compared the computation times required to solve the full QP (11) to the times required to set up and solve the reduced QP (15). All calculations were carried out with the interior-point solver QPIP [17] and repeated with the activeset solver QPAS [17] (6250 - 40 full QPs with QPIP and QPAS each, 6250 • 40 reduced QPs with QPIP and QPAS each $)^{1}$. Results are summarized in Figure $2(a, b)$ by plotting cumulative distribution functions $c d f\left(t_{\mathrm{MPC}}\right)$. The $c d f\left(t_{\mathrm{MPC}}\right)$ is the fraction of values that are equal or less than the step time $t_{\text {MPC }}$.

Approximately $25 \%$ of the QPs are detected to be unconstrained by the proposed method. Therefore, no optimization problem needs to be solved at all in these cases. Instead, the optimal control law of the unconstrained case (13) can

\footnotetext{
${ }^{1}$ All QPs were implemented in Matlab. The simulations are carried out on an Intel i5-3570 CPU with 8Gb RAM running Suse Linux with Kernel 3.11.10-7.
}

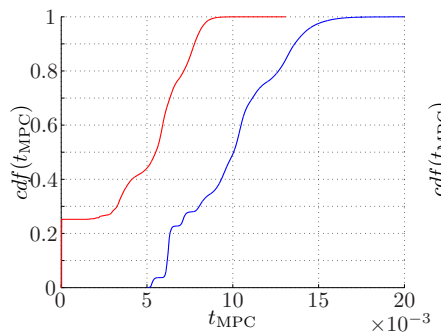

(a) QPIP

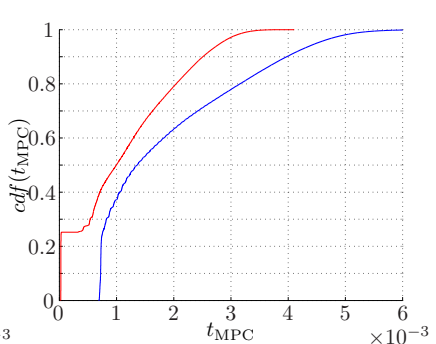

(b) QPAS

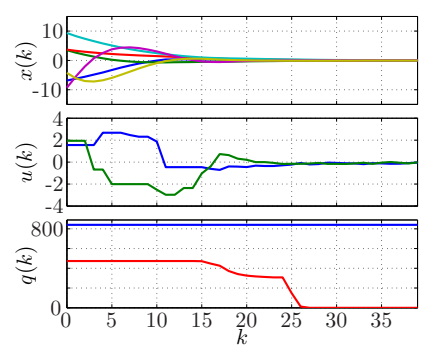

(c)

Fig. 2: Results of the simulation study: cumulative distribution functions (cdf) of the step times achieved by solving the reduced QP (red) and achieved by solving the full QP (blue) for $2.5 \cdot 10^{5}$ different quadratic programs with the QPIP solver (a) and QPAS solver (b). (c) states $x(k)$ (upper), inputs $u(k)$ (middle) and number of constraints (bottom) of the reduced QP for the trajectory resulting from one of the 6250 random chosen initial points. The black dashed line shows the number of constraints of the full QP.

be applied immediately (line 11-13 of Algorithm 1). Since the control law can be provided very quickly in these cases, the leftmost shoulder of the red curves in Figure 2(a,b) is obtained. Moreover, about $45 \%$ (resp. 38\%) of the reduced QPs are solved faster than the fastest time required to solve the full QP with the QPIP solver (resp. QPAS solver). This can be seen as follows: Consider a line parallel to the ordinate that cuts the abscissa at the smallest computation time achieved by the MPC variant without constraint removal (blue curve). The intersection of this line with the cdf resulting for solving the reduced QP (red curve) yields the value we are looking for. We claim the results shown in Figure 2(a,b) clearly indicate that the proposed method results in an acceleration for both solvers.

Consider Figure 2(c) next, which shows the states of the system, the inputs and the constraints of the reduced quadratic program for one of the initial points. The system states converge to the RPI set $\mathbb{Z}$ since the system is stable under the tube-based control law (9) (upper plot). The number of constraints $q(k)$ of the reduced QP (red curve) and the full QP (blue curve) are shown in the lowermost plot. Clearly, $q(k)$ of the reduced quadratic program converges to zero along the trajectory. At $k=27$ the optimization problem is detected to be unconstrained and the optimal solution (13) of the unconstrained problem is applied.

The full QP has 840 constraints in this example. The number of constraints of the reduced QP varies between 0 and 474, with an average of 319.67. Thus, even in the worst case $\approx 45 \%$ of the constraints are removed. About $63 \%$ of 
the constraints are removed on average.

\section{CONCLUSIONS}

We showed how to accelerate tube-based MPC by constraint removal, a technique recently introduced for the nominal MPC case [13]. Essentially, the cost function, which is a Lyapunov function under the usual assumptions, is used to detect, in the present time step, which constraints will be inactive in the subsequent time step. By removing inactive constraints from the MPC problem, the computational effort needed to solve it can be reduced. We showed that considerable reductions result with an example of moderate size.

We stress constraint removal does not require a specific QP solver, but it can easily be integrated into existing tubebased MPC implementations. We also note that no special requirements need to be imposed for constraint removal to be applicable. Finally, it is worth mentioning that it merely is convenient but not necessary to remove the states from the MPC problem formulation.

Future research has to address the identification of active constraints. These constraints can be integrated as equality constraints into the reduced quadratic program and, roughly speaking, define a linear subspace that contains the optimal solution. Moreover, extensions to nonlinear systems are obviously worth investigating.

\section{ACKNOWLEDGMENT}

Support by the Deutsche Forschungsgemeinschaft (DFG) under grant MO 1086/11-1 is gratefully acknowledged.

\section{APPENDIX}

The state space matrices of the example system read

\begin{tabular}{|c|c|c|c|}
\hline \multirow{6}{*}{$A=$} & $\left(8.34 \cdot 10^{-1}\right.$ & $-2.04 \cdot 10^{-1}$ & 0.00 \\
\hline & $1.15 \cdot 10^{-1}$ & $9.87 \cdot 10^{-1}$ & 0.00 \\
\hline & 0.00 & 0.00 & $8.82 \cdot 10^{-1}$ \\
\hline & 0.00 & 0.00 & 0.00 \\
\hline & 0.00 & 0.00 & 0.00 \\
\hline & 0.00 & 0.00 & 0.00 \\
\hline \multirow{6}{*}{$B=$} & $\left(1.15 \cdot 10^{-1}\right.$ & 0.00 & \\
\hline & $7.38 \cdot 10^{-3}$ & 0.00 & \\
\hline & $2.94 \cdot 10^{-2}$ & 0.00 & \\
\hline & 0.00 & $2.94 \cdot 10^{-2}$ & \\
\hline & 0.00 & $1.09 \cdot 10^{-1}$ & \\
\hline & 0.00 & $\left.1.43 \cdot 10^{-2}\right)$ & \\
\hline
\end{tabular}
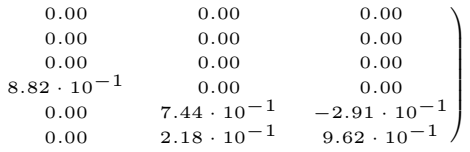

\section{REFERENCES}

[1] D.Q. Mayne, M.M. Seron, and S.V. Raković, "Robust model predictive control of constrained linear systems with bounded disturbance," Automatica, vol. 41, pp. 219-224, 2005.

[2] E. C. Kerrigan and J. M. Maciejowski, "Feedback min-max model predictive control using a single linear program: robust stability and the explicit solution," International Journal of Robust and Nonlinear Control, vol. 14, no. 4, pp. 395-413, 2004.

[3] D.Q. Mayne, E.C. Kerrigan, and P. Falugi, "Robust model predctive control: advantages and disadvantages of tube-based methods," in Proceedings of the 18th IFAC World Congress, Milano, Italy, 2011.

[4] A. Bemporad and M. Morari, "Robust model predictive control: A survey," in Robustness in identification and control, ser. Lecture Notes in Control and Information Sciences, A. Garulli and A. Tesi, Eds. Springer London, 1999, vol. 245.

[5] D.Q. Mayne, J.B. Rawlings, C.V. Rao, and P.O.M. Scokaert, "Constrained model predictive control: Stability and optimality," Automatica, vol. 36, pp. 789 - 814, 2000.

[6] P. Scokaert, J. Rawlings, and E. Meadows, "Discrete-time stability with perturbations: Application to model predictive control," Automatica, vol. 33, no. 3, pp. 463-470, 1997.
[7] D. M. Raimondo, D. Limon, M. Lazar, L. Magni, et al., "Min-max model predictive control of nonlinear systems: A unifying overview on stability," European Journal of Control, vol. 15, no. 1, pp. 5-21, 2009.

[8] W. Langson, I. Chryssochoos, S. Rakovic, and D. Mayne, "Robust model predictive control using tubes," Automatica, vol. 40, no. 1, pp. 125-133, 2004.

[9] D. Mayne, E. Kerrigan, E. Van Wyk, and P. Falugi, "Tube-based robust nonlinear model predictive control," International Journal of Robust and Nonlinear Control, vol. 21, no. 11, pp. 1341-1353, 2011.

[10] M. Jost and M. Mönnigmann, "Accelerating online MPC with partial explicit information and linear storage complexity in the number of constraints," in Proceedings of the European Control Conference 2013, Zurich, Switzerland, 2013, pp. 35-40.

[11] - "Accelerating model predictive control by online constraint removal," in Proceedings of the Conference on Decision and Control 2013, Florence, Italy, 2013, pp. 5764-5769.

[12] M. Jost, G. Pannocchia, and M. Mönnigmann, "Accelerating linear model predictive control by constraint removal," submitted, 2014.

[13] — "Online constraint removal: accelerating MPC with a Lyapunov function," Automatica, 2014, (accepted).

[14] D. S. Bernstein, Matrix Mathematics - Theory, Facts and Formulas. Princeton University Press, USA, 2009.

[15] S.V. Raković, E.C. Kerrigan, K.I. Kouramas, and D.Q. Mayne, "Invariant approximation of the minimal robust positivley invariant set,' IEEE Transactions on Automatic Control, vol. 50, no. 3, pp. 406-410, 2005

[16] E. G. Gilbert and K. T. Tan, "Linear systems with state and control constraints: The theory and application of maximal output admissible sets," IEEE Transactions on Automatic Control, vol. 36, no. 9, pp. 1008-1020, 1991

[17] D. A. Wills, QPC - Quadratic Programming in C, School of Electrical Engineering and Computer Science, University of Newcastle, 2009 [Online]. Available: http://sigpromu.org/quadprog/index.html 